\title{
Do surfaces hold the key to COVID clots?
}

T he discovery that some vaccines against SARS-CoV-2 can induce dangerous - and in extremely rare cases, fatal - blood clotting temporarily threw some COVID-19 vaccination programmes into disarray. Amidst the clamour, it was often overlooked that blood clotting is also one of the hazards of infection by the coronavirus itself, where it poses a considerably greater risk than that from vaccines.

It's likely this shared problem is no coincidence. The vaccines associated with the (very small) clotting risk are those that use a viral vector for the antigen: specifically, a disabled form of the adenovirus that causes the common cold. And that's no surprise in itself, because viral and bacterial infection have long been recognized as a potential cause of thrombogenesis (clot formation) stemming from the inflammatory immune response. But it's not really understood how the problem arises. If we had better answers, that might not only suggest ways of ameliorating the dangers of COVID-19 but could also point to strategies for suppressing this troubling side-effect of vaccines.

It seems that the key might lie in the physico-chemical processes that lead to self-assembly of the proteins involved in blood clotting. Thrombosis is an unwelcome consequence of the otherwise vital process of coagulation in the blood: the mechanism that stops bleeding from injuries. This involves a protein called fibrinogen, which is soluble in blood plasma. In normal coagulation, the enzyme thrombin cleaves fibrinogen into a polymerizable form that self-assembles into fibrous structures called fibrin. These entangle and create docking points for platelet and red blood cells, together forming a clot.

The clotting process is somehow triggered when SARS-CoV-2 binds to the membrane of cells in the tissues lining the airways and lungs, initiating an immune response ${ }^{1}$.
The concentration of fibrinogen in the blood of infected people can be much greater than usual ${ }^{2}$, just as it can for pneumonia induced by the flu virus H1N1.

Fibrinogen is a dimeric polypeptide in which each monomer is composed of three distinct chains, linked end to end by a central peptide unit (the $\mathrm{E}$ domain). The two free ends of the chains are capped by a second unit (D domain) attached to a folded region called the $\alpha \mathrm{C}$ domain.

Some years ago, Tunc et al. reported that the $\mathrm{D}$ and $\mathrm{E}$ units are strongly attracted to a hydrophobic surface, so that fibrinogen absorbs in a flat, stretched-out configuration. On a hydrophilic surface, in contrast, the terminal $\alpha \mathrm{C}$ domains are strongly bound, making the fibrinogen dimer clump up in globular form.

Rafailovich and co-workers ${ }^{4}$ showed that these absorbed conformations can initiate the formation of fibrin fibrils even without thrombin present. On a hydrophobic surface the $\alpha \mathrm{C}$ domains dangle freely from the ends of the polypeptide, and can bind those same units on other fibrinogen molecules - leading to the deposition of supramolecular layers, and eventually, the growth of fibrils. More recently, Rafailovich's group found support for this idea in studies of fibrinogen aggregation and fibril formation on surfaces with different hydrophobicity ${ }^{5}$. The fibrils appear readily on hydrophobic polystyrene surfaces with a water contact angle of around $90^{\circ}$, but not on more hydrophilic polyvinyl pyridine (contact angle $56^{\circ}$. In both cases a comparable amount of fibrinogen is absorbed on the surfaces, but its conformation in the latter instance isn't conducive to supramolecular assembly of the fibrils. What's more, fibril formation can be reduced by blocking the $\alpha \mathrm{C}$ domains using antibodies.

Those results may have important implications for reducing the risk of thrombosis induced by the surfaces

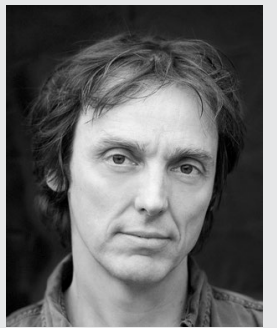

Philip Ball

of implants such as stents. But in work in preparation - presented at the 5th International Fibrinogen Research Society Workshop, Geneva, 15-16 June 2021 - Rafailovich and colleagues have shown that the same clot-initiation process can be triggered in cells infected by $\mathrm{H} 1 \mathrm{~N} 1$ and adenovirus, and they think something similar might happen with SARS-CoV-2. Their hypothesis is that the inflammatory response provoked by the viruses might damage the epithelial cells that line blood vessel walls in a way that creates conditions promoting strong fibrinogen absorption and fibrin formation - even though the virus cannot attach directly to epithelial cells themselves, since they lack the required cell-surface receptors ${ }^{6}$.

The idea will need to be tested with further studies in vitro and in vivo. If it checks out, it might be possible to identify agents that could suppress fibrin self-assembly (for example, by inactivating the $\alpha \mathrm{C}$ domains), both after viral infection and after vaccination. $\square$

Published online: 28 July 2021 https://doi.org/10.1038/s41563-021-01068-2

References

1. Mackman, N., Antoniak, S., Wolberg, A. S., Kasthuri, R. \& Key, N. S. Arterioscler. Thromb. Vasc. Biol. 40, 2033-2044 (2020).

2. Helms, J. et al. Intensive Care Med. 46, 1089-1098 (2020).

3. Tunc, S. et al. Colloids Surf. B: Biointerfaces $\mathbf{4 2}$, 219-225 (2005).

4. Koo, J. et al. Biomacromolecules 13, 1259-1268 (2012).

5. Zhang, L. et al. Acta Biomater. 54, 164-174 (2017).

6. Conde, J. N., Schutt, W. R., Gorbunova, E. E. \& Mackow, E. R. mBio 11, e03185-20 (2020). 